\title{
EVALUATION OF PROGNOSTIC FACTORS IN PATIENTS WITH RELAPSED HODGKIN'S LYMPHOMA UNDERGOING AUTOLOGOUS STEM CELL TRANSPLANT
}

\author{
Jhanzeb Iftikhar, Hafiz Abubakar Sarwar, Fareeha Sheikh, Mohammad Iqbal Shah, Shafquat Ali Khan, \\ Muhammad Abu Bakar, Neelam Siddiqui \\ Shaukat Khanum Memorial Cancer Hospital \& Research Center, Lahore Pakistan
}

\begin{abstract}
Objective: To identify the prognostic factors in relapsed Hodgkin's Lymphoma patients with regards to their impact on the outcome of autologous hematopoietic stem cell transplant.

Study Design: Retrospective observational study.

Place and Duration of Study: Department of Medical Oncology, Shaukat Khanum Memorial Cancer Hospital \& Research Center, Lahore, Jun 1999 to Jun 2019.

Methodology: Out of a total of 2061 Hodgkin's Lymphoma patients, 37 (1.8\%) patients with relapsed disease underwent autotransplant and were studied using the Hospital Information System. We obtained details of clinicopathological factors, treatment, and outcome. In our study, the outcome variable was event after transplant (relapse/disease progression/death).

Results: Among the 37 relapsed Hodgkin's Lymphoma patients undergoing auto-transplant, 24 (64.9\%) patients had an early relapse after first-line chemotherapy. In this early relapse group of 24 patients, $9(37.5 \%)$ remained well after auto-transplant but $15(62.5 \%)$ patients had an event. Out of 37 relapsed Hodgkin's Lymphoma patients undergoing auto-transplant, a complete response on pre-transplant imaging was observed in $24(64.9 \%)$ patients. In those 24 patients with a complete response on pre-transplant imaging, $16(66.7 \%)$ patients remained well after transplant while $8(33.3 \%)$ patients had a worse event. Twoyear progression-free and overall survival proportions were $56 \%$ and $77 \%$ respectively.

Conclusion: Complete metabolic response on pre-transplant imaging was associated with better overall survival. Adverse factors observed were initial short duration of complete response, bulky disease at relapse, variables comprising international prognostic score, B symptoms, and raised erythrocyte sedimentation rate at relapse.
\end{abstract}

Keywords: Autologous stem cell transplant, Hodgkin's lymphoma, Prognostic factors.

How to Cite This Article: Iftikhar J, Sarwar HA, Sheikh F, Shah MI, Khan SI, Bakar MA, Siddiqui N. Evaluation of Prognostic Factors in Patients with Relapsed Hodgkin's Lymphoma Undergoing Autologous Stem Cell Transplant. Pak Armed Forces Med J 2021; 71(5): 1833-1838.

Doi: https://doi.org/10.51253/pafmj.v71i5.4132

This is an Open Access article distributed under the terms of the Creative Commons Attribution License (https://creativecommons.org/licenses/by-nc/4.0/), which permits unrestricted use, distribution, and reproduction in any medium, provided the original work is properly cited.

\section{INTRODUCTION}

Hodgkin's Lymphoma (HL) is a potentially curable disease with a favorable overall survival (OS) of $88 \%$ at 5 years and $82 \%$ at 15 years ${ }^{1,2}$. More than $90 \%$ of the patients with Hodgkin's Lymphoma are cured with induction chemotherapy alone such as Adriamycin based chemotherapy containing adriamycin, bleomycin, vinblastine, and dacarbazine (ABVD) or in combination with radiation therapy (XRT). However, 20-30\% of patients have relapsed or primary refractory disease. Standard of care for patients with relapsed or refractory disease is a combination salvage chemotherapy followed by autologous stem cell transplant (ASCT) ${ }^{3}$, in chemosensitive patients. High dose chemotherapy followed by autotransplant has shown superior eventfree survival (EFS) in relapsed Hodgkin's Lymphoma

Correspondence: Dr Jhanzeb Iftikhar, Medical Oncology Shaukat Khanum Memorial Cancer Hospital \& Research Center, Lahore Pakistan Received: 20 Apr 2020; revision received: 10 May 2020; accepted: 14 May 2020 patients when compared with salvage chemotherapy alone. Retrospective studies have shown that disease status at relapse, chemosensitivity to salvage chemotherapy, and duration of initial complete response (CR) are significant prognostic factors for the outcome of ASCT $^{4-6}$.

Pre-transplant identification of relapsed or refractory Hodgkin's Lymphoma patients who have a poor prognosis despite autotransplant would provide an opportunity to offer investigational therapies to higher-risk patients. Newer agents such as brentuximab vedotin (BV) and checkpoint inhibitors (e.g. pembrolizumab and nivolumab) are currently being utilized to optimize response on pre-ASCT positron emission tomography/computed tomography (PET/CT) scan 7-10. However, due to financial implications, these medications are not currently available in Pakistan. To achieve complete metabolic response (CMR) post-transplant, an alternative approach is to intensify the transplant conditioning regimen but this is at the expense of 
increased treatment-related toxicity including secondary malignancies.

The purpose of this study was to identify the prognostic indicators in patients with relapsed HL undergoing ASCT and to analyze the survival outcomes.

\section{METHODOLOGY}

This retrospective observational study was conducted on HL patients diagnosed and treated between June 1999 to June 2019 at Shaukat Khanum Memorial Cancer Hospital and Research Center, Lahore. Out of a total of $2061 \mathrm{HL}$ patients reporting at SKMCH, $37(1.8 \%)$ patients with relapsed disease underwent ASCT. The data was extracted using the electronic hospital information system (HIS) after obtaining approval from the Institutional Review Board (reference no: EX-05-04-19-05 dated 26-04-2019). We obtained details of clinic-pathological factors, radiological features, treatment, and outcome. A questionnaire was designed and initially, data was collected on a questionnaire. Non-probability consecutive sampling technique was used. In this study, our outcome variable was an event after a transplant that was defined as relapse/ disease progression/death.

Inclusion Criteria: Chemotherapy responsive relapsed disease with a partial or complete response on PET/ $\mathrm{CT}$, age 16-40 years, normal renal/hepatic profile and along with adequate cardiac (ejection fraction $>50 \%$ ) and pulmonary function (diffusion lung capacity $>50 \%$ predicted).

Exclusin Criteria: Non-consenting patients were excluded.

Written informed consent was taken from all patients before undergoing auto-transplant. BEAM protocol was used as conditioning regimen[ BCNU $\left(300 \mathrm{mg} / \mathrm{m}^{2}\right.$ i.v. on day -6$)$, etoposide $\left(200 \mathrm{mg} / \mathrm{m}^{2}\right.$ i.v. on day-5,-4,-3,-2), cytarabine $\left(200 \mathrm{mg} / \mathrm{m}^{2}\right.$ i.v. twice daily on day-5,-4,-3,-2) and melphalan $\left(140 \mathrm{mg} / \mathrm{m}^{2}\right.$ i.v. for day -1)]. All patients received granulocyte colonystimulating factor (G-CSF) $5 \mathrm{mg} / \mathrm{kg}$ SQ bid, continuing until ANC $>1000 / \mathrm{uL}$ for at least 3 consecutive days. Patients were radiologically assessed before ASCT, on day+90 after ASCT, and thereafter if clinically indicated. The median duration of follow up was 24 months (range 1-153).

Relapse was differentiated from primary progression by a 3 months remission period after completion of the first-line treatment. Refractory disease included patients with stable disease (SD) or progressive disease
(PD) after primary therapy. Early relapse was defined as disease recurrence or progression within 12 months of induction treatment and late relapse as disease recurrence or progression after 12 months of induction treatment. The patients were staged according to international response criteria for lymphoma ${ }^{11}$. Progression-free survival (PFS) was measured in months from date of transplant or date of last salvage chemotherapy to date of relapse after transplant/censored date. Overall survival was calculated in months from the date of transplant until the date of the last follow up for alive patients. In OS, the event of interest was death for which date of death was collected.

Statistical analysis was carried out using SPSS20. Percentages (proportion) were used for categorical variables while mean and standard deviation were used for continuous variables. Chi-square and Fisher exact tests were used for the bivariate analysis of categorical variables. Kaplan-Meier survival curves were used to assess OS and PFS of patient's cohort. Statistical significance was defined as a two-tailed $p$-value $\leq 0.05$. Variables related to disease biology, patient characteristics, and international prognostic score (IPS) were examined.

\section{RESULTS}

Out of the 37 patients who underwent autologous stem cell transplant for relapsed or refractory HL, 24 $(64.9 \%)$ were males and $13(35.1 \%)$ females. The mean age at initial diagnosis was $22.92 \pm 6.6$ years; however, the mean age at the time of transplant was $26 \pm 6$ years. At initial diagnosis, $26(70.3 \%)$ patients had stage IV disease; B symptoms were present in 34 (91.9\%) patients and $25(67.6 \%)$ patients had extra-nodal involvement as shown in the Table-I. Most of the patients received Adriamycin containing regimen 35 (94.6\%). Radiotherapy was done in $10(27 \%)$ patients. The median duration of first complete response (CR) after firstline chemotherapy had been 4 months (1-127); of these, $24(64.9 \%)$ patients had an early relapse while 13 (35.1\%) patients had a late relapse. The time interval between initial diagnosis and date of transplant ranged from 10-141 months with a median of 20 months. Out of 37 patients, $24(64.9 \%)$ patients had CR on pre-transplant imaging while 13 (35.1\%) had PR (Table-ll).

After the first-line chemotherapy, 24 (64.9\%) patients at the initial presentation had an early relapse and $13(35.1 \%)$ patients had a late relapse. In the early relapse group, $9(37.5 \%)$ patients remained in remission while $15(62.5 \%)$ patients had relapsed or progressive disease after auto-transplant. Out of 13 (35.1\%) patients 
Table-I: Patients characteristics at the initial diagnosis.

\begin{tabular}{l|c}
\hline Characteristics & $\mathbf{n}=\mathbf{3 7}(\%)$ \\
\hline Age (Years) & $22.92 \pm 6.6$ \\
\hline Mean \pm SD & $3(8.1)$ \\
\hline Stage & $4(10.8)$ \\
\hline I & $4(10.8)$ \\
\hline II & $26(70.3)$ \\
\hline III & $35(94.6)$ \\
\hline Histopathology & $2(5.4)$ \\
\hline Classical Hodgkin's Lymphoma & $3(8.1)$ \\
\hline Nodular Lymphocytic predominant & $34(91.9)$ \\
Hodgkin's Lymphoma & $21(56.8)$ \\
\hline B-Symptoms & $16(43.2)$ \\
\hline Absent & $19(51.4)$ \\
\hline Present & $18(48.6)$ \\
\hline None Marrow Involvement & $12(32.4)$ \\
\hline Involved & $25(67.6)$ \\
\hline Bulky Disease & \\
\hline Absent & \\
\hline Present & \\
\hline Extranodal Involvement & \\
\hline Absent & Present \\
\hline
\end{tabular}

in the late relapse group, $10(7.6 .9 \%)$ patients had disease remission post ASCT while $3(23.1 \%)$ patients had disease relapse after transplant. Out of 13 (35.1\%) patients having partial response on pre-transplant PET/CT scan, $10(76.9 \%)$ had an event after auto-transplant and $3(23.1 \%)$ patients having PR did not have any event. However, out of the $24(64.9 \%)$ patients having CMR on pre-transplant PET/CT scan, $16(66.7 \%)$ patients remained well after ASCT and $8(33.3 \%)$ patients had an adverse outcome after transplant (Figure-1).

Two-year progression-free survival (PFS) and overall survival (OS) proportions were $56 \%$ and $77 \%$ respectively. Progression-free survival at 2 years for early relapse was $42 \%$ and for late relapse, $88 \%$; however, overall survival at 2 years for early relapse was $73 \%$ and for late relapse $85 \%$. Our analysis also suggests that the disease status of CMR on PET/CT post salvage pre-transplant was associated with a better outcome. Progression-free survival at 2 years for the patients having complete response was $64 \%$ and for the patients with partial response, it was $40 \%$. However, OS at 2 years for CR was $86 \%$, and for PR it was $64 \%$.

Raised serum lactate dehydrogenase (LDH), bulky disease, B symptoms, low lymphocyte count, and low albumin were also shown to adversely affect the outcome. In our study advanced stage, male sex,
Table-II: Patients characteristics at relapse versus event status.

\begin{tabular}{|c|c|c|c|}
\hline Characteristics & $\begin{array}{l}\text { No Events } \\
19(51.4 \%)\end{array}$ & $\begin{array}{c}\text { Events } \\
18(48.6 \%)\end{array}$ & $\begin{array}{c}p- \\
\text { value }\end{array}$ \\
\hline \multicolumn{4}{|l|}{ Gender } \\
\hline Male & $13(54.2)$ & $11(45.8)$ & \multirow{2}{*}{0.64} \\
\hline Female & $6(46.2)$ & $7(53.8)$ & \\
\hline \multicolumn{4}{|l|}{ Stage (Relapse) } \\
\hline I & $1(100)$ & - & \multirow{5}{*}{0.19} \\
\hline II & $1(50)$ & $1(50)$ & \\
\hline III & $3(75)$ & $1(25)$ & \\
\hline IV & $10(62.5)$ & $6(37.5)$ & \\
\hline Primary progression & $4(28.6)$ & $10(71.4)$ & \\
\hline \multicolumn{4}{|l|}{ Hemoglobin } \\
\hline Low & $4(33.3)$ & $8(66.7)$ & \multirow{2}{*}{0.13} \\
\hline Normal & $15(60)$ & $10(40)$ & \\
\hline \multicolumn{4}{|l|}{ Albumin } \\
\hline Low & $2(13.3)$ & $13(86.7)$ & \multirow{2}{*}{0.001} \\
\hline Normal & $17(77.3)$ & $5(22.7)$ & \\
\hline \multicolumn{4}{|c|}{ White Blood Cell Count } \\
\hline Normal & $15(62.5)$ & $9(37.5)$ & \multirow{2}{*}{0.06} \\
\hline Raised & $4(30.8)$ & $9(69.2)$ & \\
\hline \multicolumn{4}{|l|}{ Lymphocyte Count } \\
\hline Low & $2(13.3)$ & $13(86.7)$ & \multirow{2}{*}{0.001} \\
\hline Normal & $17(77.3)$ & $5(22.7)$ & \\
\hline \multicolumn{4}{|l|}{ B Symptoms } \\
\hline Absent & $17(60.7)$ & $11(39.3)$ & \multirow{2}{*}{0.06} \\
\hline Present & $2(22.2)$ & $7(77.8)$ & \\
\hline \multicolumn{4}{|l|}{ Bulky Disease } \\
\hline Absent & $18(60)$ & $12(40)$ & \multirow{2}{*}{0.04} \\
\hline Present & $1(14.3)$ & $6(8.7)$ & \\
\hline \multicolumn{4}{|c|}{ Extra nodal Involvement } \\
\hline Absent & $10(66.7)$ & $5(33.3)$ & \multirow{2}{*}{0.12} \\
\hline Present & $9(40.9)$ & $13(59.1)$ & \\
\hline \multicolumn{4}{|c|}{ Erythrocyte Sedimentation Rate } \\
\hline Normal & $13(86.7)$ & $2(13.3)$ & \multirow{2}{*}{0.001} \\
\hline Raised & $6(27.3)$ & $16(72.7)$ & \\
\hline \multicolumn{4}{|l|}{ Lactate Dehydrogenase } \\
\hline Normal & $15(78.9)$ & $4(21.1)$ & \multirow{2}{*}{0.001} \\
\hline Raised & $4(22.2)$ & $14(77.8)$ & \\
\hline \multicolumn{4}{|c|}{ Eastern Cooperative Oncology group Performance Status } \\
\hline 1 & $8(72.7)$ & $3(27.3)$ & \multirow{3}{*}{0.06} \\
\hline 2 & $11(50)$ & $11(50)$ & \\
\hline 3 & - & $4(100)$ & \\
\hline Pre-Transplant Positr & n Emission & nography Sc & \\
\hline Partial response & $3(23.1)$ & $10(76.9)$ & 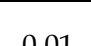 \\
\hline Complete response & $16(66.7)$ & $8(33.3)$ & 0.01 \\
\hline Prior Radiotherapy & & & \\
\hline No & $12(44)$ & $15(55.6)$ & 0.27 \\
\hline Yes & $7(70)$ & $3(30)$ & 0.27 \\
\hline
\end{tabular}

low hemoglobin $(\mathrm{Hb})$, and extra-nodal involvement at the time of relapse after frontline chemotherapy were not statistically significant. Out of the 37 patients undergoing ASCT, 9 patients died. Of those 9 patients, 7 deaths were disease relapse deaths and 2 were due 
to transplant-related mortality (TRM). TRM day 100 was $5.4 \%$; the cause of death in these cases was sepsis. Salvage chemotherapy followed by ASCT provided good outcomes comparable with published data. Disease status of CMR post salvage pre-transplant was associated with better OS. Other adverse factors observed were short duration of initial CR, high tumor burden, variables making IPS (albumin, lymphopenia), B symptoms, and raised erythrocyte sedimentation rate
(ESR) at the time of relapse (Figure-2).

\section{DISCUSSION}

The current treatment of choice for relapsed Hodgkin's Lymphoma patients is high dose chemotherapy followed by autotransplant. British National Lymphoma Investigation (BNLI) and the German Hodgkin's Study Group (GHSG)/European Bone Marrow Transplantation Group (EBMT) (HDR-1 protocol) sho-

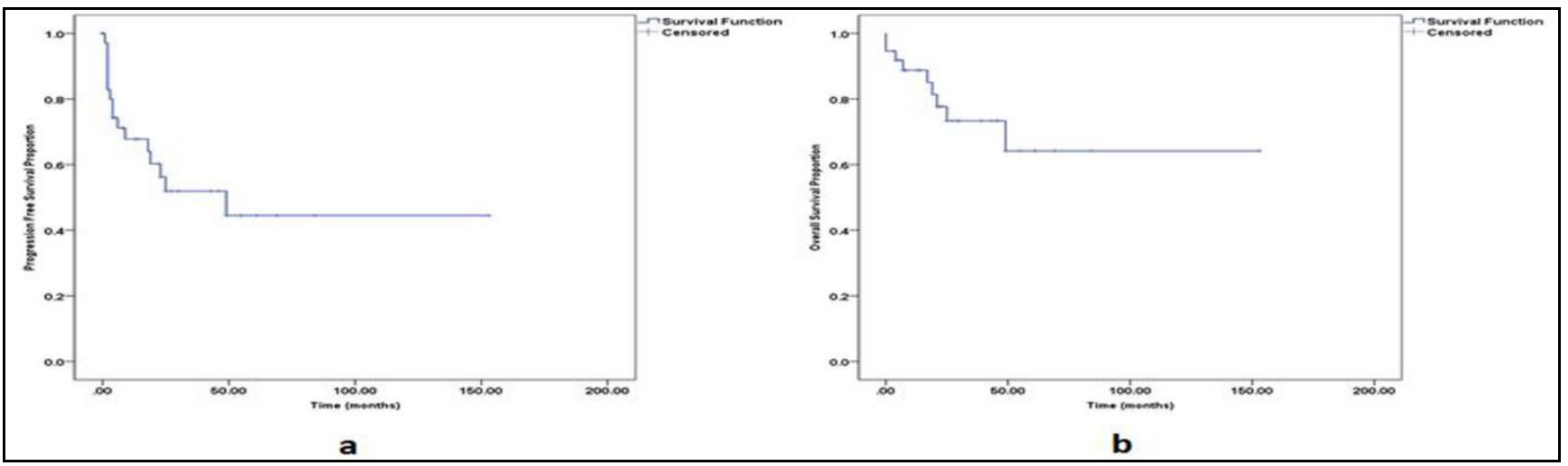

Figure-1 (a\&b): Kaplan Meier curve. (a) Progression free survival proportion of relapsed Hodgkin's lymphoma patients undergoing ASCT, (b) Overall survival proportion of relapsed Hodgkin's lymphoma patients undergoing ASCT.
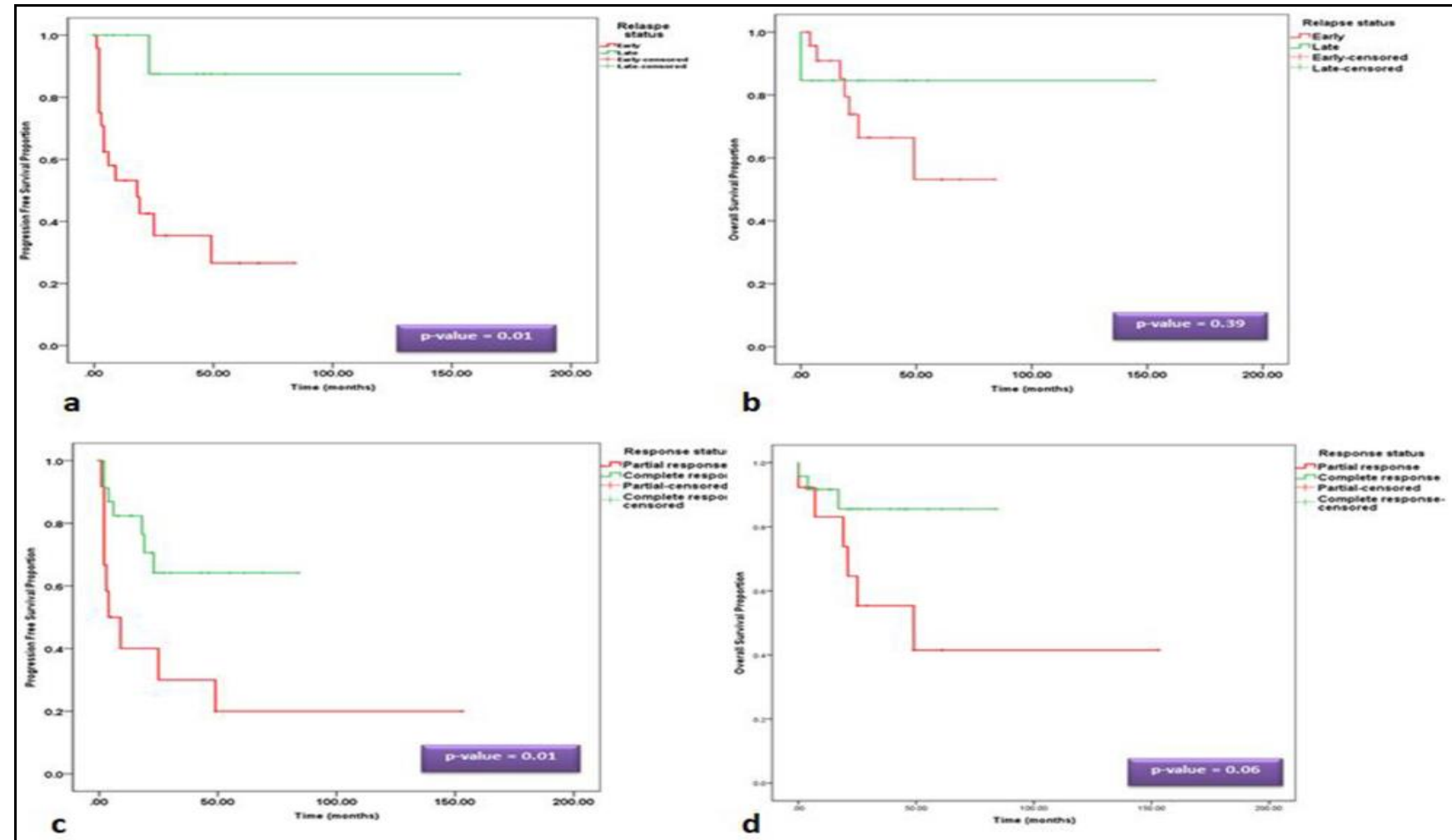

Figure-2(a, b, c \& d): Kaplan Meier curve. (a) Progression free survival proportion in comparison to early and late relapse, (b) Comparison of the overall survival proportion in patients with early versus late relapse, (c) Progression free survival proportion in comparison to partial and complete response on pre-transplant imaging, (d) Comparison of the overall survival proportion in patients with early versus late response on pre-transplant imaging. 
wed improved outcome in relapsed HL patients treated with high-dose chemotherapy ${ }^{12,13}$. This improvement was observed in both early and late relapse in terms of freedom from the second failure ${ }^{13}$. These studies showed ASCT as an option better than conventional salvage chemotherapy. However, disease recurrence after transplant occurs in a significant proportion of patients. In this context, an effective evaluation of prognostic factors at the time of the first relapse is needed. This evaluation can help direct the most appropriate investigational therapies and the development of experimental approaches in patients predicted to have a poor outcome after transplant.

In our study, we have interpreted disease, patient, and transplant-related prognostic factors with regards to outcome after ASCT in a group of 37 patients autografted for HL relapsing after frontline therapy. Two years PFS and OS proportions were $56 \%$ and $77 \%$ respectively. In our analysis, the short duration of initial CR after first-line chemotherapy, the presence of PETpositive disease pre-transplant, and bulky disease at relapse became indicators of an adverse outcome after autotransplant. Most of the studies have already shown that chemosensitivity before ASCT is a favorable prognostic indicator. However, the need to achieve a CMR on pre-transplant PET/CT has not been fully studied warranting the need for future prospective trials.

Bierman et al. conducted a study in a series of 128 relapsed HL patients treated homogenously with CBV protocol. It was summed up that mediastinal disease, poor functional status, and failure of two or more chemotherapy lines were indicators of unfavorable endresults post ASCT, with a 4-year failure-free survival of only $10 \%$ in those patients failing with two or more protocols ${ }^{14}$. Reece et al, identified four prognostic groups in a group of 58 patients treated with the transplant. The prognostication was done based on the presence of extranodal disease, B symptoms, and a short initial complete response. With one risk factor, progression-free survival at 3 years was 100\%, $40 \%$ in those with two risk factors, and $0 \%$ in patients with three risk factors ${ }^{15}$. The group of the City of Hope evaluated patients autografted with the CBV protocol or the combination of TBI- cyclophosphamide, and etoposide. It was concluded that prior radiotherapy, more than 2 prior chemotherapy protocols, and extra-nodal disease at autotransplant were indicators of an adverse outcome after transplant ${ }^{16}$. Similarly, in a study on 119 relapsed or refractory HL patients autografted with the CBV regimen or the TBI-cyclophosphamide and etoposide protocol, the prognostication of patients was done based on a combination of B symptoms, presence of lymph nodes $>2 \mathrm{~cm}$, and bone marrow or pulmonary involvement at ASCT with 4-year event-free survivals of $85 \%$ in patients with no adverse prognostic factors compared with $41 \%$ in patients with one bad prognostic fractor ${ }^{17}$.

Likewise, the French cooperative group (GELA) and GHSG study identified short initial CR and extranodal involvement along with the clinical stage and anemia at relapse as prognostic factors 18,19 . The Memorial Sloan-Kettering Cancer Center analyzed 65 relapsed or refractory patients treated with two biweekly cycles of ifosfamide, carboplatin, and etoposide. B symptoms, extra-nodal disease, and short initial CR were identified as independent risk factors. Event-free survival rates of $83 \%$ and $10 \%$ were observed in patients with $0-1$ and 3 risk factors respectively ${ }^{20}$.

\section{LIMITATION OF STUDY}

The limitations of our study include a small sample size and a relatively short follow-up period. However, as Hodgkin's Lymphoma relapse mostly occurs in the first 2 years after therapy so we can rely on two-year data. These prognostic factors need to be confirmed by observing these patients for a longer period of follow up. There was a nonavailability of PET/CT scan in 3 patients. This was mainly due to the inclusion of patients from the pre-PET era and therefore the rate of the refractory disease may be artificially higher. This could partly explain the rather good outcomes in refractory HL patients in our cohort.

\section{CONCLUSION}

The results of our study suggest that it is possible to form a prognostic factor score to guide treatment for relapsed HL patients undergoing auto-transplant. The aim should be to achieve complete metabolic response on post salvage pre-transplant PET/CT. Exploration of newer agents in patients with less than partial response to salvage chemotherapy before proceeding with auto-transplant especially in patients not able to have more intensified conditioning regimens should be considered in prospective (randomized control trials and cohort) studies.

\section{Conflict of Interest: None.}

\section{Authors' Contribution}

JI: Idea of research and manuscript writing, HAS: Study design, analysis and result writing, FS: Acquisition of data, MIS: Data collection, SAK: Manuscript writing, MAB: Statistical analysis.

\section{REFERENCES}

1. Ansell SM. Hodgkin lymphoma: update on diagnosis, risk stratification, and management. Am J Hematol 2016; 91(4): 434-442. 


\section{Relapsed Hodgkin's Lymphoma}

2. Engert A, Raemaekers J. Treatment of early-stage Hodgkin lymphoma. Semin Hematol 2016; 53(3): 165-170.

3. Von-Tresckow B. Treatment of relapsed and refractory Hodgkin lymphoma. Semin Hematol 2016; 53(3): 180-185.

4. Adams HJ, Kwee TC. Prognostic value of pretransplant FDGPET in refractory/relapsed Hodgkin lymphoma treated with autologous stem cell transplantation: systematic review and meta-analysis. Ann Hematol 2016; 95(5): 695-706.

5. Bröckelmann PJ, Müller $\mathrm{H}$, Casasnovas $\mathrm{O}$, Hutchings $\mathrm{M}$, von Tresckow B. Risk factors and a prognostic score for survival after autologous stem-cell transplantation for relapsed or refractory Hodgkin lymphoma. Ann Oncol 2017; 28(6): 1352-1358.

6. Moskowitz AJ, Schöder H, Gavane S, Thoren KL, Fleisher M, Yahalom J, et al. Prognostic significance of baseline metabolic tumor volume in relapsed and refractory Hodgkin lymphoma. Blood 2017; 130(20): 2196-2203.

7. Mottok A, Steidl C. Biology of classical Hodgkin lymphoma: implications for prognosis and novel therapies. Blood 2018; 131(15): 1654-1665.

8. Chen R, Zinzani PL, Fanale MA, Armand P. Phase II study of the efficacy and safety of pembrolizumab for relapsed/refractory classic Hodgkin lymphoma. J Clin Oncol 2017; 35(19): 2125.

9. Chen R, Palmer JM, Martin P, Tsai N, Kim Y, Chen BT, et al. Results of a multicenter phase II trial of brentuximab vedotin as second-line therapy before autologous transplantation in relapsed/refractory Hodgkin lymphoma. Biol Blood Marrow Transplant 2015; 21(12): 2136-2140.

10. Moskowitz CH, Nademanee A, Masszi T, Agura E, Holowiecki J, Abidi $\mathrm{MH}$, et al. Brentuximab vedotin as consolidation therapy after autologous stem-cell transplantation in patients with Hodgkin's lymphoma at risk of relapse or progression (AETHERA): a randomised, double-blind, placebo-controlled, phase 3 trial. Lancet 2015; 385(9980): 1853-1862.

11. Cheson BD, Pfistner B, Juweid ME, Gascoyne RD, Specht L, Horning SJ, et al. Revised response criteria for malignant lymphoma. J Clin Oncol 2007; 25(5): 579-586.

12. Linch D, Goldstone AH, McMillan A, Chopra R, Hudson GV. Dose intensification with autologous bone-marrow transplantation in relapsed and resistant Hodgkin's disease: results of a BNLI randomised trial. Lancet 1993; 341(8852): 1051-1054.
13. Schmitz N, Pfistner B, Sextro M, Sieber M, Carella AM, Haenel $\mathrm{M}$, et al. Aggressive conventional chemotherapy compared with high-dose chemotherapy with autologous haemopoietic stemcell transplantation for relapsed chemosensitive Hodgkin's disease: a randomised trial. Lancet 2002; 359(9323): 2065-2071.

14. Linch D, Goldstone AH, McMillan A, Chopra R, Hudson GV. Dose intensification with autologous bone-marrow transplantation in relapsed and resistant Hodgkin's disease: results of a BNLI randomised trial. Lancet 1993; 341 (8852): 1051-1054.

15. Reece DE, Connors JM, Spinelli JJ, Barnett MJ, Fairey RN, Klingemann HG, et al. Intensive therapy with cyclophosphamide, carmustine, etoposide \pm cisplatin, and autologous bone marrow transplantation for Hodgkin's disease in first relapse after combination chemotherapy. Blood 1994; 83(5): 1193-1199.

16. Nademanee A, O'donnell MR, Snyder DS, Schmidt GM, Parker PM, Stein AS, et al. High-dose chemotherapy with or without total body irradiation followed by autologous bone marrow and /or peripheral blood stem cell transplantation for patients with relapsed and refractory Hodgkin's disease: results in 85 patients with analysis of prognostic factors. Blood 1995; 85(5): 1381-1390.

17. Horning SJ, Chao NJ, Negrin RS, Hoppe RT, Long GD, Hu WW, et al. High-dose therapy and autologous hematopoietic progenitor cell transplantation for recurrent or refractory Hodgkin's disease: analysis of the Stanford University results and prognostic indices. Blood 1997; 89(3): 801-813.

18. Brice $\mathrm{P}$, Bouabdallah $\mathrm{R}$, Moreau $\mathrm{P}$, Divine $\mathrm{M}$, Andre $\mathrm{M}$, Aoudjane M, et al. Prognostic factors for survival after high-dose therapy and autologous stem cell transplantation for patients with relapsing Hodgkin's disease: analysis of 280 patients from the French registry. Bone Marrow Transplant 1997; 20(1): 21-25.

19. Josting A, Franklin J, May M, Koch P. New prognostic score based on treatment outcome of patients with relapsed Hodgkin's lymphoma registered in the database of the German Hodgkin's lymphoma study group. J Clin Oncol 2002; 20(1): 221-230.

20. Schmitz N, Pfistner B, Sextro M, Sieber M, Carella AM, Haenel $\mathrm{M}$, et al. Aggressive conventional chemotherapy compared with high-dose chemotherapy with autologous haemopoietic stemcell transplantation for relapsed chemosensitive Hodgkin's disease: A randomised trial. Lancet 2002; 359(9323): 2065-2071. 